\title{
Enfermedad pulmonar intersticial y crepitantes. Algunas reflexiones sobre sus características y localización
}

\author{
Interstitial Lung Disease and Crackles. Some Reflections on their Characteristics and Location
}

\author{
Francisco José Fernández-Fernández, Eugenia Ameneiros Lago \\ Servicio de Medicina Interna. Hospital Arquitecto Marcide. SERGAS. Ferrol
}

\section{Sr. Director,}

Hemos leído con interés la excelente revisión "Una mirada general a las enfermedades pulmonares intersticiales y una específica a la fibrosis pulmonar idiopática" publicada recientemente en su revista por Marcos et al ${ }^{1}$. Como bien señalan los autores, la presencia de crepitantes, que suelen ser secos y en las bases pulmonares, puede sugerir la presencia de una enfermedad pulmonar intersticial. Asimismo, existe una importante demora entre el inicio de los síntomas y el diagnóstico final de la fibrosis pulmonar idiopática.

Nos gustaría hacer algunas reflexiones, desde el punto de vista de nuestra práctica clínica habitual, sobre algunas características de los crepitantes y su distribución que puede ayudar en el diagnóstico diferencial. Desde el punto de vista de la terminología, los crepitantes son ruidos anormales adventicios, discontinuos, que se superponen a los ruidos normales. Es frecuente que se refleje en la historia clínica la presencia de "crepitantes secos" o "crepitantes húmedos". Estos son 2 de los 4 términos descriptivos que utilizó Laënnec como hallazgos de la auscultación con su estetoscopio cilíndrico de madera. Esta clasificación como "secos o húmedos", que la mayoría de nosotros usamos en numerosas ocasiones, no se recomienda por algunos autores². Una clasificación posterior los ha diferenciado en crepitantes finos y gruesos. Los gruesos son de tono más grave y tienden a ser menos reproducibles que los finos, modificándose con la tos. Suelen ser el resultado de la acumulación de secreciones en los bronquios. Los crepitantes finos son de tono relativamente alto, inspiratorios y no se modifican con la tos. Muchos investigadores han explorado la potencial especificidad de ciertas características de los crepitantes para entidades específicas, con varios escenarios en los que los hallazgos de la auscultación podrían orientar hacia un diagnóstico3. En la sarcoidosis, los crepitantes son menos frecuentes que en la fibrosis pulmonar idiopática. Así, en un estudio ${ }^{4}$ publicado en la década de los 70, en el que se intentaba correlacionar los hallazgos clínicos, radiológicos y patológicos, únicamente un 20\% de pacientes con sarcoidosis u otras enfermedades gra- nulomatosas presentaban crepitantes en la auscultación frente a un $60 \%$ con neumonías intersticiales. Esta baja incidencia de crepitantes en la sarcoidosis ha sido también descrita por otros autores. Así, en la sarcoidosis, podemos encontrarnos con un paciente con una alteración radiológica muy expresiva y una auscultación relativamente "limpia". Al contrario, en la asbestosis los crepitantes pueden aparecer precozmente, con radiografía de tórax y pruebas de función respiratoria normales ${ }^{5,6}$. En sujetos con exposición a las fibras de asbesto, la auscultación de crepitantes puede ser un signo temprano de asbestosis.

Más frecuente, en nuestra práctica clínica habitual, es que los pacientes con enfermedad pulmonar intersticial difusa tengan factores de riesgo vascular 0 una cardiopatía conocida asociada. Ya que los crepitantes finos de la fibrosis son similares a los causados por insuficiencia cardiaca ${ }^{5}$, en pacientes con miocardiopatía, una fibrosis pulmonar puede pasar fácilmente desapercibida y el paciente ser sometido a un tratamiento diurético excesivo y prolongado. En estos pacientes, la auscultación de crepitantes en los campos pulmonares anteriores puede ser un hallazgo útil que nos indique la presencia de una fibrosis pulmonar oculta asociada ${ }^{7}$.

\section{Bibliografía}

1. Marcos PJ, Montero C, Otero González I. Una mirada general a las enfermedades pulmonares intersticiales y una específica a la fibrosis pulmonar idiopática. Galicia Clin 2013;74(4):13-22.

2. Piirilä $P$, Sovijärvi ARA. Crackles: recording, analysis and clinical significance. Eur Respir J 1995; 8(12): 2139-2148.

3. Pasterkamp H, Kraman SS, Wodicka GR. Respiratory sounds. Advances beyond the stethoscope. Am J Respir Crit Care Med 1997;156(3):974-987.

4. Epler GR, Carrington CB, Gaensler EA. Crackles (Rales) in the interstitial pulmonary diseases. Chest 1978;73(3):333-339.

5. Murphy RL Jr, Gaensler EA, Holford SK, Del Bono EA, Epler G. Crackles in the early detection of asbestosis. Am Rev Respir Dis 1984; 129(3):375-379.

6. Shirai F, Kudoh S, Shibuya A, Sada K, Mikami R. Crackles in asbestos workers: auscultation and lung sound analysis. Br J Dis Chest 1981;75(4):386-396.

7. Sud M, Barolet A, McDonald M, Floras JS. Anterior crackles: A neglected sign?. Can J Cardiol 2013. doi: 10.1016/j.cjca.2012.11.014. [Epub ahead of print]. 